\title{
ARE MEASURED GROUND CONTROL POINTS STILL REQUIRED IN UAV BASED LARGE SCALE MAPPING? ASSESSING THE POSITIONAL ACCURACY OF AN RTK MULTI-ROTOR PLATFORM.
}

\author{
L. Teppati Losè̀ ${ }^{1,}$, F. Chiabrando ${ }^{1}$, F. Giulio Tonolo ${ }^{1}$ \\ ${ }^{1}$ LabG4CH, Department of Architecture and Design (DAD) - Politecnico di Torino Viale Mattioli 39, 10125 Torino (Italy) \\ (lorenzo.teppati, filiberto.chiabrando, fabio.giuliotonolo)@polito.it
}

Commission I, ICWG I/II

KEY WORDS: UAVs, SfM, RTK, PPK, Direct Georeferencing, 3D Mapping

\begin{abstract}
:
The estimate of External Orientation (E.O.) parameters for a block of images is a crucial step in the photogrammetric pipeline and the most demanding in terms of required time and human effort, both during the fieldwork and post-processing phases. Different researchers developed strategies to minimize the impact of this phase. Despite the achievement of good results, it was not possible until now to completely cancel the effect of this step. However, the efforts of the researchers in these years have also been devoted to the implementation of direct photogrammetry strategies, in order to almost completely automate the E.O. of the photogrammetric block. These new approaches were made possible also thanks to the latest developments of commercial UAVs, especially in terms of the installed GPS/GNSS (Global Positioning System/Global Navigation Satellite System) hardware. The aim of this manuscript is to evaluate the different perspectives and issues connected with the deployment of a UAV (Unmanned Aerial Vehicle) equipped with a multi-frequency GPS/GNSS receiver. Starting from the considerations mentioned above and leveraging previous works based on a fixed-wing platform, the focus of this contribution is the assessment of the real performances of an RTK multi-rotor platform addressing several questions. Is it possible to generate added-value products with centimetre 3D accuracies without measuring any ground control point? Which are the operational requirements to be taken into account in the planning phase? Are consolidated UAV mapping operational workflows already available to enable a robust direct georeferencing approach?
\end{abstract}

\section{INTRODUCTION}

The deployment of UAVs for metric survey applications can be considered as a standard approach nowadays, and different field of applications are benefiting from the latest developments of this technology. The enhancement of SfM (Structure from Motion) algorithms, in parallel with the continuous development of the commercial platforms, led to standardizing and automated approaches both in the phases of data acquisition and processing. However, in the overall UAVs photogrammetric pipeline, a crucial point still requires a high manual and human intervention: the task connected with the block E.O. solution. This step is usually solved using GCPs (Ground Control Points) with known coordinates, entailing different implications, both in the phases of fieldwork acquisition and data-processing. During the acquisition phase is thus necessary to measure a set of control points, generally adopting traditional topographic techniques such as Total Station or GNSS measurements. It is sometimes possible to use well-recognizable natural features of the surveyed site as control points, but the most consolidated and orthodox approach foreseen the use of artificial codified target that needs to be homogenously distributed along with the site and after that measured.

The adoption of GCPs is a crucial element in terms of time optimisation also during the processing phase. Control points need to be located and checked in the acquired images; this operation is still the less automated and the more timeconsuming in the overall photogrammetric processing pipeline. Moreover, it is also essential to consider the impact that GCPs has in the estimation of I.O. (Interior Orientation) parameters of the used camera if an a-priori calibration is not available.

\footnotetext{
* Corresponding author
}

As reported in the following paragraphs, several researchers addressed their efforts in the direction of optimizing this phase, adopting different strategies and solutions. In this framework, this contribution aims at exploiting the possibilities offered from one of the latest UAV platforms released from the Chinese company DJI (SZ DJI Technology Co., Ltd). This platform, described in paragraph 2.1, allows to adopt RTK (Real-time kinematic), NRTK (Network Real-Time Kinematic) and PPK (Post-Processed Kinematic) approaches, thanks to the onboard multi-frequency multi-constellation GNSS receiver. Such approaches can drastically reduce the time needed for E.O. phase without affecting the accuracy of the final 3D model achieved through an SfM approach. It is thus necessary to carefully validate the results achievable with these approaches and the operational practices that need to be followed to acquire and process the data accurately. The research presented in this contribute is derived also by a previous work reported in (Chiabrando, Giulio Tonolo, \& Lingua, 2019). In this work, a similar strategy was set up to validate a PPK approach using a fixed-wing commercial platform, while the present contribute is focused on a multi-rotor one.

Although this platform became available on the market more than a year ago, few researches have been conducted and published on its use and validation for mapping purposes. Some experiences are shown in the works undertaken by (Peppa, Hall, Goodyear, \& Mills, 2019; Taddia, Stecchi, \& Pellegrinelli, $2019,2020)$. This fact is probably ascribable to two main aspects: the high cost (more than $5 \mathrm{k} €$ ) of the platform and the absence of a DJI built-in solution for the automatic processing of the acquired GNSS data. These aspects may probably influence the diffusion of this platform among professional operators. 


\subsection{UAVs photogrammetry fields of application}

The UAVs platforms, starting from their early developments, have been a topic of high interest in the community of geomatics researchers and the possibilities connected with their use have been exploited by several authors. UAVs have thus been successfully used in several fields of application (as reported for example in Nex \& Remondino, 2014). These field of application varies from agriculture, archaeology, architecture, emergency management, environmental monitoring, forestry applications, industrial application and traffic monitoring. Of particular success is the use of UAVs in the field of $\mathrm{CH}$ (Cultural Heritage) documentation. This success can be related to some winning elements provided by the introduction of UAVs in the traditional survey pipeline adopted in this field, i.e., the rapid development of commercial platforms and onboard sensors, a decrease of cost in comparison with traditional aerial systems, the reduction of object-sensor distance, the easiness of deployment of these systems in different contexts and finally the introduction of a new unconventional low altitude aerial point of view on $\mathrm{CH}$ objects or sites. Several types of research have been carried out in the last years concerning this topic (Bolognesi, Furini, Russo, Pellegrinelli, \& Russo, 2015; Georgopoulos, Oikonomou, Adamopoulos, \& Stathopoulou, 2016; Sauerbier \& Eisenbeiss, 2010) however, some issues are still open and need to be further investigated. One of the topics that is still under development is the solution of the E.O. phase for a photogrammetric block, that is a time-consuming operation.

\subsection{E.O. solution. Issue, strategies}

For the reasons mentioned above, several Geomatics studies focused their attention on research topics connected with both practical issues of field deployment of UAVs such us flight planning, camera orientation, balance evaluation between number and position of ground control points, typology of imaging sensors, etc., and data processing (e.g. camera calibration, image matching, reliability of onboard sensors, etc.). Most of the aforementioned studies were mainly aimed at automating the whole process to reduce human intervention and to speed up the phases of the photogrammetric workflow.

E.O. is the phase of photogrammetric workflow with the lowest degree of automatization of the whole process, while the impact of the other processing steps is transferred to the computational resources of the computer thanks to the improvements of the algorithms performances of these approaches in the last years. As already reported, different researchers developed strategies to minimize the impact of this phase in the overall photogrammetric process, focusing on a combination of two main strategies:

- the enhancement of the flight planning phase (with the support also provided by the integration of oblique images). As reported for example in (Aicardi et al., 2016; Chiabrando, Lingua, Maschio, \& Teppati Losè, 2017; Nesbit \& Hugenholtz, 2019);

- the optimisation of the number and position of ground control points. As reported for example in (AgüeraVega, Carvajal-Ramírez, \& Martínez-Carricondo, 2017; Martínez-Carricondo et al., 2018; Tahar, 2013).

Despite the good results that could be achieved and an overall optimisation of these phases it was not possible until now to completely cancel this step.

\subsubsection{Direct georeferencing with PPK and RTK approaches}

Direct georeferencing approaches can be deployed to skip or minimizing tasks connected to GCPs. The idea behind these approaches is to automate the E.O. phase of the photogrammetric block. This approach is enabled by the presence of a high precision GNSS receiver with RTK capabilities onboard the UAVs platform. The data collected and registered from this receiver allow to adopt RTK, NRTK or PPK approaches; the first two approaches are exploited directly on the field while the PPK in a second phase of the processing. Until the release of Phantom 4 RTK, few alternative commercial solutions able to be successfully used for direct georeferencing purposes were available on the market. Research experiences in this sense were generally conducted adopting self-built or customised solutions. The efforts of the researchers were thus addressed also in designing, testing and calibrating these hardware solutions and their deployment in the field.

Some examples can be found in (Benassi et al., 2017; Gabrlik, 2015; Rabah, Basiouny, Ghanem, \& Elhadary, 2018). Several advantages can be achieved by adopting a direct georeferencing approach, mainly, it is possible to eliminate the control point positioning and measuring phase on the field and their processing in the subsequent processing phases. This can be traduced in a time-saving approach and consequently also in a reduction of the cost both for the fieldwork activities and processing steps. Moreover, this solution can become a winning element for the mapping of dangerous or inaccessible areas, where is not possible to position and measure control points.

\section{ACQUISITION STRATEGIES}

The site chosen to perform the tests presented in this manuscript is Castellazzo di Caluso (Torino Province, Piemonte Region, north-western Italy), an archaeological site that hosts the remains of a medieval fortification dating back to the XIII century. The place was chosen due to its peculiar morphological configuration developed on three levels (as shown in Figure 1 b). The whole area of the site was imaged during the aerial photogrammetric survey enabling the generation of a complete 3D model of the archaeological/architectural remains. Moreover, the three levels configuration of the site guarantees the perfect set-up to test the investigated approaches over an area with a high elevation difference. Before the planning and execution of the different flights it was necessary to set up a set of control points to assess the accuracy of the proposed photogrammetric approaches. A collection of 17 artificial codified targets was thus homogeneously distributed on the area of interest as showed in Figure 1 a.

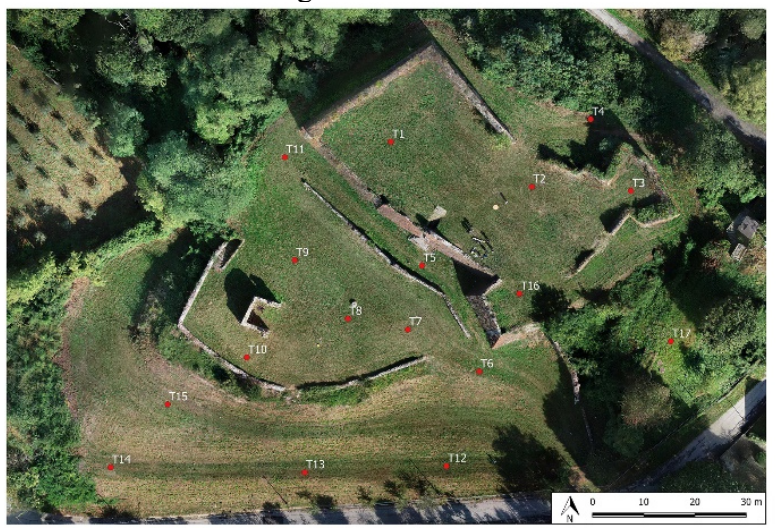

Figure 1 (a) Distribution of the control points measured on the test site (red dots) 


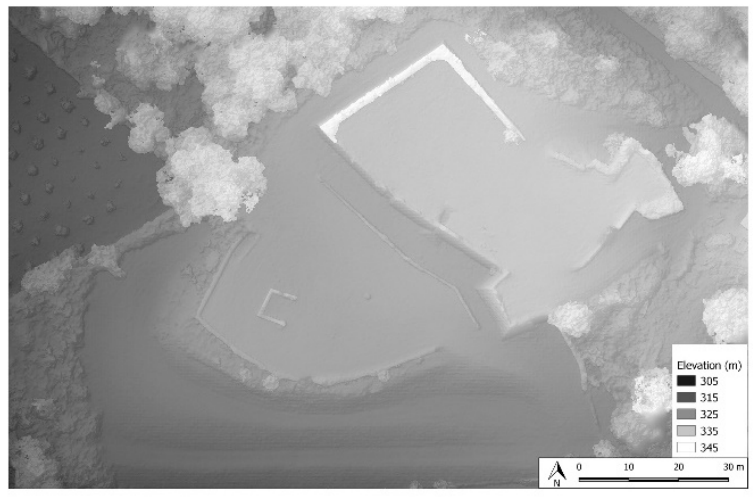

Figure 1 (b) DSM of the surveyed area.

The targets were then measured through a traditional topographic approach using a GNSS receiver (Geomax Zenith 35 ) in NRTK mode thanks to the correction provided by the SPIN 3 GNSS service ${ }^{1}$. The precision of the measurements of the control points was around $\pm 1.5 \mathrm{~cm}$ for the horizontal component and around $\pm 2 \mathrm{~cm}$ for the vertical component. This set of 17 points was then used as for the tests reported before.

\subsection{The UAV platform}

The current paper is based on tests carried out with a DJI Phantom 4 RTK, embedding an RTK GNSS module that can provide real-time accurate data on the camera positions. The Phantom 4 RTK can manage estimation and recording of the position in three main ways: i) storing the GNSS information to be used for PPK approach, ii) connecting with a GNSS Networked Transport of RTCM via Internet Protocol (NTRIP) for an NRTK approach or iii) connecting with a GNSS master station provided by DJI (D-RTK2 mobile station) that works as a reference point on the ground) for an RTK approach.

The Phantom 4 RTK is similar to the previous Phantom 4 Pro: it mounts a mechanical shutter camera equipped with a 1" CMOS 20 MP sensor. The main difference of the Phantom 4 RTK resides in the multi-frequency and multi-constellation GNSS receiver for which DJI claims a position accuracy of $1.5 \mathrm{~cm}+1$ ppm for the vertical component and $1 \mathrm{~cm}+1 \mathrm{ppm}$ for the horizontal component ${ }^{2}$. Moreover, this platform is compatible with the DJI D-RTK 2 mobile station ${ }^{3}$, a GNSS receiver able to provide real-time differential corrections to the company's platforms

The combination of Phantom 4 RTK and D-RTK2 station (Figure 2) allows then to implement different acquisition strategies on the field depending on the positioning approach chosen from the users.

\footnotetext{
${ }^{1}$ https://www.spingnss.it/spiderweb/frmIndex.aspx

2 All the specifications of the Phantom 4 RTK are available at https://www.dji.com/uk/phantom-4-rtk/info\#specs

${ }^{3}$ Specification at https://www.dji.com/uk/d-rtk-2/info\#specs
}

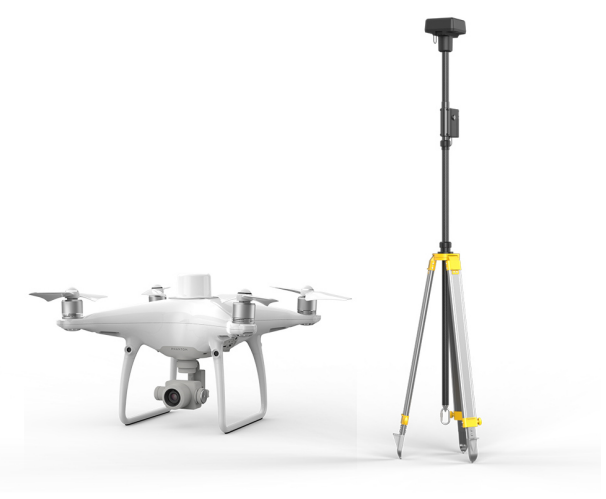

Figure 2 The DJI Phantom 4 RTK (left) and the D-RTK 2 mobile station (right).

The onboard GNSS receiver is a multi-constellation (GPS, GLONASS, Galileo, Beidou) multi-frequency one. It has a sampling rate for the raw data of $5 \mathrm{~Hz}$ and data are recorded in RINEX (Receiver Independent Exchange Format) format, version 3.03.

Another important characteristic of this platform is the possibility to store all the imagery metadata (exposure time, camera attitude, GNSS antenna offset, etc.) in a dedicated file called timestamp.

Given the three modalities in which the phantom can estimate and record its position (i, ii and iii), five different acquisition strategies can be achieved:

- Traditional approach: the flight is performed adopting the conventional approach, and the positioning is demanded to the onboard GNSS receiver but without any correction applied.

- PPK approach: the acquisition is again performed traditionally, but GNSS data recorded by a base station are necessary to apply PPK during the postprocessing operation. Both a receiver on the field or a CORS (Continuously Operating Reference Station) station can be used.

- $\quad$ NRTK approach: thanks to the NTRIP service in this approach, the UAVs is connected via the internet through the radio controller device and can download the needed corrections from a network of geodetic stations.

- D-RTK2 approach: in this case, the DJI base station is used as a reference base station to perform the images using a PPK approach. The main difference with the second approach is that the station is also connected with the UAV and is thus possible to obtain information also on the relative position between these two entities. Without knowing the coordinates of the base station, a PPK approach is necessary after the acquisition.

- D-RTK2 with point of known coordinates: this approach is similar to the previous one but can be considered as an RTK approach. The base station is positioned on a point of known coordinates and corrections are directly transmitted to the UAV.

All the five acquisitions strategy were tested in the selected test site and will be described and discussed in the following paragraphs. The dataset acquired for the standard processing can be used also in a PPK approach if some conditions are applied: i.e. the presence of a GNSS receiver working as ground station or the possibility of using the data collected derived from a CORS stations. 


\subsection{Flight panning and configuration}

In the test site of the Castellazzo di Caluso different flights were achieved with the Phantom 4 RTK however, it was decided to follow a single flight scheme, pre-programmed thanks to the dedicated mobile application DJI Pilot. A nadiral acquisition was thus programmed with the following characteristics: flight lines with East-West direction, high overlap (80\% longitudinal and $70 \%$ lateral), medium flight height 40 meters. An overall number of 92 images was acquired with a GSD (Ground Sampling Distance) of $1.1 \mathrm{~cm}$, covering an area of around $15.000 \mathrm{~m}^{2}$. The acquisition scheme of the flights performed in the test area is reported in the following Figure 3.

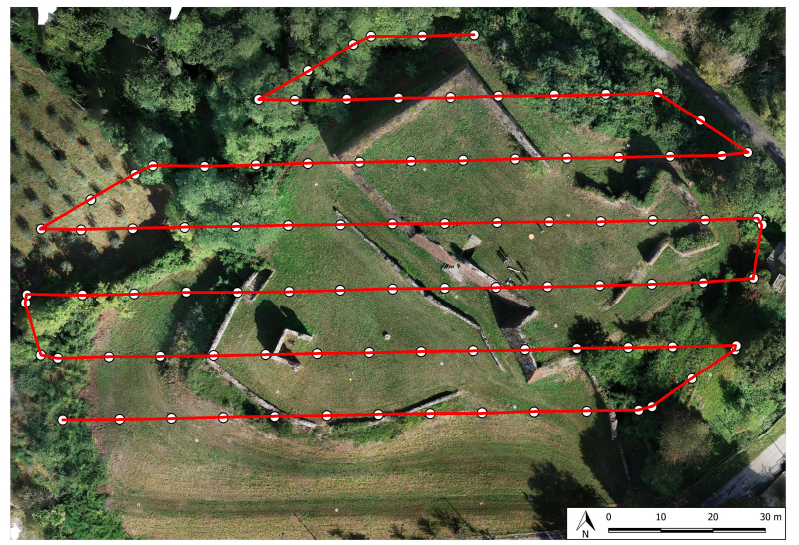

Figure 3 Flight plan for Castellazzo area. Flight lines in red and camera positions highlighted with white dots.

Four acquisitions were initially planned during the fieldwork: 1 for standard and PPK approach, 1 with the NRTK approach and 2 using the D-RTK 2 station. The solution adopting the D-RTK 2 as the ground station is partially different because it needs to be connected with the UAVs during the field operation and partially works differently if compared with a third part GNSS receiver.

\subsection{Three acquisition strategies}

Among the different acquisitions performed during the fieldwork only two datasets will be considered in this manuscript, while the approaches using the D-RTK2 antenna will be exploited in future researches.

All the data were collected after the measurement of the prepositioned targets, used as $(\mathrm{G}) \mathrm{CP}$ in subsequent processing phases.

The first dataset considered is Dataset A, that was processed both following a standard procedure (described in section 3.1) and a PPK approach (described in section 3.3) using the data retrieved from a CORS of the Italian geodetic network.

Dataset B was considered to exploit the possibilities connected with the NRTK approach. This approach requires the setup of specific parameters on the DJI pilot application that allows the connection between the UAVs platform and NTRIP network, to download the corrections in real-time. The processing of this approach is described in paragraph 3.4 .

\section{DATA PROCESSING}

The data collected on the field were processed adopting different strategies and using the well-known photogrammetric suite Agisoft Metashape (previously Photoscan) version 1.6.2. The aim of this paper is to evaluate the impact of different georeferencing strategies on the first phases of the photogrammetric processing, and thus only these steps were considered. No further analyses were carried out on the influence of the same parameters on the photogrammetric derived products, such as point cloud, mesh, DEM (Digital Elevation Model), etc.

\subsection{Dataset A - Standard data processing}

In a first step, the first dataset acquired was processed following the traditional photogrammetric approach, i.e. image matching, tie points extraction, BBA (Bundle Block Adjustment) thanks to the use of control points.

Among the 17 measured control points it was decided to select 11 points to be used as GCPs and 6 as CPs (Check Points). The main difference between these two types of points is that GCPs are used in the solution of the BBA phase, while CPs are left outside of the process and used as independent accuracy checks. The points to be used as CPs were selected in order to present a homogenous distribution on the overall surveyed area especially considering the differences in the elevation component of the archaeological site.

The RMSe (Root Mean Square error) on both GCPs and CPs for the standard processing of Dataset A is reported in Table 2, after some consideration on the estimation of I.O. parameters.

\subsection{The importance of camera calibration}

As well documented in the literature, a crucial step of the whole photogrammetric pipeline is the camera calibration, i.e. the estimation of I.O. parameters. Part of this work was thus dedicated also to this aspect, mainly because DJI provides the platforms of Phantom 4 RTK family with a camera calibration certificate. More specifically, information about some of the I.O. parameters (focal length, principal point, $k 1, k 2, k 3, p 1$ and p2) are directly embedded in the EXIF file (Exchangeable image file format). Dataset A was thus processed following two different approaches for camera calibration, one with the certificate provided from DJI and the other with a selfcalibration approach. The values estimated for the different I.O. parameters are reported in the following Table 1 .

\begin{tabular}{|c|c|c|}
\hline & Pre-calibrated & Self-calibration \\
\hline $\mathbf{f}$ & 3635.190 & 3620.157 \\
\hline $\mathbf{k 1}$ & -0.264 & -0.266 \\
\hline $\mathbf{k 2}$ & 0.111 & 0.114 \\
\hline $\mathbf{k 3}$ & -0.038 & -0.043 \\
\hline $\mathbf{k 4}$ & 0.000 & 0.008 \\
\hline $\mathbf{c x}$ & 2.480 & -4.262 \\
\hline $\mathbf{c y}$ & 9.690 & 6.526 \\
\hline $\mathbf{p 1}$ & 0.000 & -0.001 \\
\hline $\mathbf{p 2}$ & -0.001 & 0.000 \\
\hline b1 & $/$ & -0.350 \\
\hline b2 & $/$ & 0.311 \\
\hline
\end{tabular}

Table 1 I.O. parameters derived from the calibration certificate provided by DJI (left) and parameters estimated through a selfcalibration approach (right)

Except for the presence of $b 1$ and $b 2$ parameters (affinity or skew), the differences between the other parameters are quite limited. However, the impact of these small differences on the accuracy of the final photogrammetric model must not be underestimated. In Table 2 the RMSe values of the same set of 
$\mathrm{GCPs} / \mathrm{CPs}$ derived from the processing of Dataset A adopting a pre-calibration or self-calibration approach are reported.

\begin{tabular}{|l|l|l|c|c|}
\cline { 2 - 5 } \multicolumn{1}{c|}{} & \multicolumn{1}{c|}{$\begin{array}{c}\text { RMSe } \\
\text { X (m) }\end{array}$} & $\begin{array}{c}\text { RMSe } \\
\text { Y (m) }\end{array}$ & $\begin{array}{c}\text { RMSe } \\
\text { Z (m) }\end{array}$ & $\begin{array}{c}\text { RMSe } \\
\text { TOT (m) }\end{array}$ \\
\cline { 2 - 5 } \multicolumn{1}{c|}{} & \multicolumn{4}{c|}{ Pre-calibration } \\
\hline 11 GCPs & 0.023 & 0.025 & 0.039 & 0.051 \\
\hline 6 CPs & 0.029 & 0.023 & 0.038 & 0.057 \\
\hline & \multicolumn{4}{|c|}{ Self-calibration } \\
\hline 11 GCPs & 0.010 & 0.008 & 0.010 & 0.016 \\
\hline 6 CPs & 0.023 & 0.012 & 0.024 & 0.035 \\
\hline
\end{tabular}

Table 2 RMSe on GCPs and CPs for standard processing of Dataset A adopting pre-calibration and self-calibration approaches.

A good solution of I.O. have a high impact on the metric accuracy of the final model, as clearly visible in Table 2 . It seems that the calibration certificate provided from DJI, as already underlined by (Taddia et al., 2020), presents some incongruences and according to these preliminary evaluations, a self-calibration approach should be preferred. The impact of camera calibration is crucial also in PPK and RTK approaches, especially for dataset composed only from nadiral images. As well reported in literature (e.g. Bolkas, 2019; Forlani et al., 2018; Honkavaara, 2002; Taddia et al., 2020) in case of direct georeferencing approaches for nadiral dataset it is necessary to use at least one GCP, in order to obtain a good accuracy of I.O. parameters and thus of the overall model. Adopting PPK or RTK approaches with only nadiral images and without GCPs can lead to a wrong estimation of camera calibration parameters, especially concerning the focal length, resulting in a significant error in the elevation component.

\subsection{Dataset A - PPK processing}

To complete the PPK processing different conditions must be met. First, all the information relative to the performed flight need to be available and stored in the proper format. In this case, this information is stored in a RINEX file which contains the GNSS observation recorded during the flight allowing to determine the platform position a-posteriori with a centimetre level accuracy. The second type of data needed is the timestamp, created in real-time during the flight and containing several information, i.e. the acquisition time of each image (in GPS time) and the offset information between APC (Antenna Phase Centre) and CMOS sensor centre. The latter information is derived by a software solution that allows to combine the offset information measured during the production of the platform (available in the firmware) from camera centre and the APC position on the aircraft body (in $\mathrm{x}, \mathrm{y}$ and $\mathrm{z}$ components) with the yaw, pitch and roll values acquired by the IMU (Inertial Measurement Unit) sensor during the flight. The second element that is needed to complete the PPK processing is the availability of GNSS observations recorded at the same time of the flight to be used as base station data to estimate the positional correction. Both a geodetic GNSS receiver used in the field, a CORS station or a virtual station created interpolating data derived from different CORS stations can be used as base station. For this paper, it was decided to use the data derived from the nearest CORS station located in Crescentino (CRSN) and part of the SPIN 3 GNSS service. The CORS station of Crescentino is located around $20 \mathrm{~km}$ SouthEast of Caluso.

Finally, even if not mandatory (but suggested when possible) also the broadcasted ephemeris files were included in the processing of the observation to increase the overall accuracy of the process.
An official solution from DJI, is not yet available to carry out a PPK processing of the data derived from acquisitions performed with the Phantom 4 RTK despite being announced. Consequently, to process the data is necessary to use third commercial part or self-built solution. Different solutions were tested $^{4}$, both commercial and open-source, and it was thus decided to focus the tests on the open solution. Nevertheless, a comparison between the different processing approaches can be interesting, both in terms of accuracy and ease of use, and is programmed for future research works.

3.3.1 An opensource solution to perform the PPK of Phantom RTK data

The first step of PPK processing is connected with postprocessing of the flight GNSS data thanks to the use of the data derived from the CORS station of Crescentino. This step was achieved using the opensource solution $\mathrm{RTKLIB}^{5}$ (version 2.4.3).

This powerful opensource programs package allows to perform all the calculation needed for this operation and is also able to provide a plot of the solution (as shown for example in Figure 5 a-b). An estimation of the standard deviation of the computed solution to check the quality of the process is also provided.

The second step of PPK consists in the interpolation of the camera positions according to the PPK-corrected flight path. This is possible thanks to the timestamp of each acquired image and is performed by means of an interpolation of the coordinates of the two closest PPK positions to the time of image acquisition. In this interpolation also the APC-sensor offsets are accounted and directly applied to the new camera coordinates in terms of $\mathrm{E}, \mathrm{N}, \mathrm{Z}$ components. The interpolation between new flight path and cameras exposure time was computed using an excel pre-compiled spreadsheet created by Aerotas $^{6}$ company and that is freely distributed by its creators.

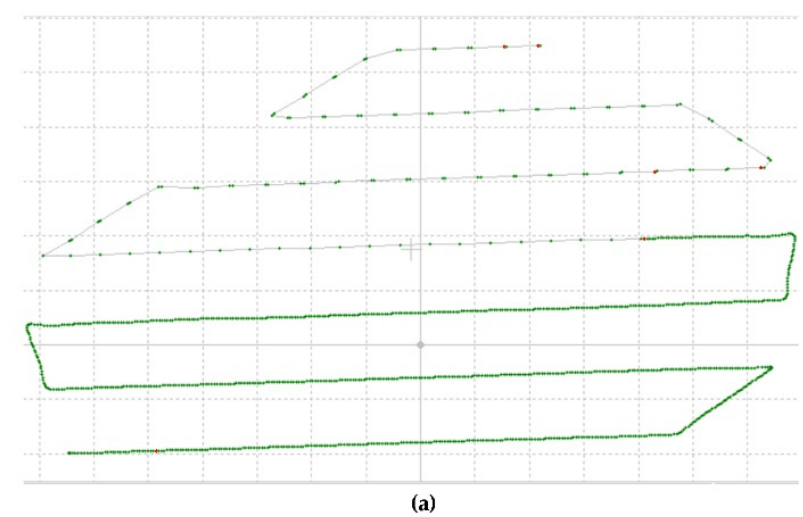

Figure 4 (a) An example of the plot derivable from RTKLIB processing. A partially successful computation. Green dots represented fixed GPS/GNSS solutions while red dots are float solutions.

\footnotetext{
${ }^{4}$ e.g. KLAUPPK or Hi-Target

${ }^{5} \mathrm{http}: / /$ www.rtklib.com/

${ }^{6} \mathrm{https}: / / \mathrm{www} \cdot$ aerotas.com/
} 




(b)

Figure 5 (b) An example of the plot derivable from RTKLIB processing. A successful computation. Green dots represented fixed GPS/GNSS solutions while red dots are float solutions.

The final step of the processing consists in the introduction of the new camera coordinates in the photogrammetric processing. This step can be achieved mainly in two modalities: using a procedure that set the new coordinates as geotag for the acquired images or importing the coordinates in the processing software. There are no specific advantages in preferring one of these two methods, and it mainly depends on the features available in the adopted photogrammetric software solution.

\subsection{Dataset B - NRTK}

The processing of the data acquired with an NRTK approach is for sure more direct and less time consuming if compared with the PPK processing; among the different possible approaches is the fastest one. In this case, camera positions are already evaluated with a high accuracy during the acquisition phase, thanks to the connection of the UAV with the CORS network (no pre-processing operations are required). For NRTK processing is thus possible to directly import the images in the employed photogrammetric software and proceed with the different steps of the pipeline. It is thus crucial to evaluate the quality and accuracy of the GNSS solution for each camera before proceeding with the processing. This information is stored in the single image metadata and can be accessed and verified. It is important to underline that in this case information about APC-sensors offsets are already considered and therefore applied to camera geotags. For the data processing the images were imported in the photogrammetric software and the information about their position were used to solve the E.O. phase.

As for Dataset A it was necessary to perform a standard processing also for Dataset B. This operation was necessary to obtain an estimation of camera position to be used for the comparisons presented in paragraph 4.2. The RMSe on GCPs and CPs for the standard processing of this dataset is reported in the following Table 3 .

\begin{tabular}{|l|c|c|c|c|}
\cline { 2 - 5 } \multicolumn{1}{c|}{} & $\begin{array}{c}\text { RMSe } \\
\text { X (m) }\end{array}$ & $\begin{array}{c}\text { RMSe } \\
\text { Y (m) }\end{array}$ & $\begin{array}{c}\text { RMSe } \\
\text { Z }(\mathbf{m})\end{array}$ & $\begin{array}{c}\text { RMSe } \\
\text { TOT }(\mathbf{m})\end{array}$ \\
\hline 11GCPs & 0.009 & 0.007 & 0.008 & 0.015 \\
\hline 6 CPs & 0.023 & 0.013 & 0.027 & 0.038 \\
\hline
\end{tabular}

Table 3 RMSe on GCPs and CPs for standard processing of dataset B.

\section{VALIDATION}

Two different methodologies were followed to validate the proposed approaches. The first is making use of the control points measured on the field while the second aims in comparing the camera centre positions obtained through direct georeferencing with the ones estimated in standard processing. For CP based accuracy assessment, the 17 points measured on the field were used as ground truth. For both PPK and NRTK approaches the same points were identified in the set of images after the phase of image matching and BBA and their coordinates were thus estimated and compared with the measured coordinates. For the second approach, the reference dataset was represented by the camera centre positions (stored in the geotag) whose coordinates were estimated in the standard photogrammetric processing (paragraph 3.1 for PPK and 3.4 for NRTK). It is essential to underline that the positions of camera centres were refined in the photogrammetric software to achieve a more accurate estimation of the sensor's attitude during the acquisition (yaw, pitch and roll) and thanks to the evaluation of camera I.O. parameters through a self-calibration approach solved in the BBA phase.

\subsection{PPK}

As well documented in the literature and as already reported in paragraph 3.2, for a dataset with only nadiral images such as the one of the Castellazzo di Caluso the implementation of a PPK and RTK process cannot be sufficient to achieve good results in terms of metric accuracy. This issue is determined by a wrong estimation of the camera's I.O. parameters. It is especially noteworthy that an incorrect evaluation of the focal length value can lead to significant errors on the elevation components of the whole photogrammetric model. Authors faced this issue also in the processing of the dataset used in this paper. A first validation of the PPK approach was conducted on a dataset that was processed without using any measured control point in the BBA phase and relying only on the information of camera position derived from the PPK processing. In Table 4 are reported the results obtained from the comparison between coordinates of the measured control points and the coordinates estimated from the PPK processing.

\begin{tabular}{|l|l|l|l|}
\cline { 2 - 4 } \multicolumn{1}{c|}{} & $\boldsymbol{\Delta X}(\mathbf{m})$ & $\boldsymbol{\Delta Y}(\mathbf{m})$ & $\Delta \mathbf{Z}(\mathbf{m})$ \\
\hline Mean & 0.002 & -0.006 & 0.727 \\
\hline Std.Dev. & \pm 0.033 & \pm 0.031 & \pm 0.108 \\
\hline
\end{tabular}

Table 4 Comparison between the coordinates of $17 \mathrm{CP}$ measured on the field and the coordinates of the same points estimated in the PPK processed without any GCP.

As is clearly visible from the figures in table 4 the choice of this approach is leading to a systematic bias $(\sim 0.7 \mathrm{~m})$ and a lower accuracy $(\sim \pm 0.11 \mathrm{~m})$ for the elevation component.

The processing was then repeated using one measured control points as GCPs in order to achieve a better solution of camera I.O. parameters estimation. The position of this point was chosen according to the strategies proposed by several authors in the literature (e.g. Martínez-Carricondo et al., 2018) and was thus a barycentric point of the surveyed area. The results are reported in Table 5.

\begin{tabular}{|l|l|l|l|}
\cline { 2 - 4 } & $\mathbf{\Delta X}(\mathbf{m})$ & $\Delta \mathbf{Y}(\mathbf{m})$ & $\Delta \mathbf{Z}(\mathbf{m})$ \\
\hline Mean & -0.003 & 0.002 & -0.023 \\
\hline Std.Dev. & \pm 0.017 & \pm 0.019 & \pm 0.031 \\
\hline
\end{tabular}

Table 5 Comparison between the coordinates of $16 \mathrm{CP}$ measured on the field and the coordinates of the same points estimated in the PPK processed with 1 GCP. 
From the data reported in Table 5 it is possible to underline an overall enhancement in the elevation accuracy, with a bias of $\sim 0.02 \mathrm{~m}$ and a similar accuracy (as expected with a PPK approach). The second analyses to validate the PPK approach was conducted by means of comparison between camera centre positions estimated with the standard processing (with several GCPs) and the coordinates of the same locations estimated in the PPK approach. The PPK dataset used in this comparison is the one where 1 GCPs is used, results are shown in Table 6.

\begin{tabular}{|l|c|c|c|}
\cline { 2 - 4 } \multicolumn{1}{c|}{} & $\boldsymbol{\Delta X}(\mathbf{m})$ & $\boldsymbol{\Delta Y}(\mathbf{m})$ & $\Delta \mathbf{Z}(\mathbf{m})$ \\
\hline Mean & -0.014 & -0.023 & -0.021 \\
\hline Std.Dev. & 0.032 & 0.018 & 0.016 \\
\hline
\end{tabular}

Table 6 Comparison between the coordinates of the 92 camera positions estimated with the standard processing (11 GCPs) and the coordinates of the same camera position estimated with the PPK processing (1 GCP).

As is possible to notice from the values in Table 6 the camera coordinates differences are in a range of few centimetres confirming the overall good solution achieved in the estimation of camera centre position in the PPK approach. The elevation bias related to the PPK approach without any GCP can be successfully solved using the one GCP approach.

\subsection{NRTK}

The same analyses conducted for the PPK validation approach were also performed for the NRTK approach (without any GCP).

The data derived from the comparison between measured and estimated coordinates of the control points are reported in the following Table 7, while in Table 8 are reported the data derived from the comparison between camera centre positions.

\begin{tabular}{|l|l|l|l|}
\cline { 2 - 4 } \multicolumn{1}{c|}{} & $\mathbf{\Delta X}(\mathbf{m})$ & $\mathbf{\Delta Y}(\mathbf{m})$ & $\Delta \mathbf{Z}(\mathbf{m})$ \\
\hline Mean & 0.054 & 0.017 & -0.068 \\
\hline Std.Dev. & 0.063 & 0.035 & 0.039 \\
\hline
\end{tabular}

Table 7 Comparison between the coordinates of the control points measured on the field and the coordinates of the same points estimated in the NRTK processing.

NRTK approach present slightly higher error if compared with the PPK approach, however still in a range that is suitable for large scale mapping application purposes at different representation scales. It still to be considered that for the NRTK approach no further intervention from the operator were required during the pre-processing of the data and also that it was possible not to use any GCPs during the processing.

\begin{tabular}{|l|l|l|l|}
\cline { 2 - 4 } \multicolumn{1}{c|}{} & $\mathbf{\Delta X}(\mathbf{m})$ & $\mathbf{\Delta Y}(\mathbf{m})$ & $\Delta \mathbf{Z}(\mathbf{m})$ \\
\hline Mean & 0.013 & -0.002 & -0.004 \\
\hline Std.Dev. & 0.072 & 0.036 & 0.046 \\
\hline
\end{tabular}

Table 8 Comparison between the coordinates of the 92 camera centre (flight 2 ) estimated with the standard processing (11

$\mathrm{GCP}$ ) and the coordinates of the same camera position estimated in the NRTK processing (no GCP).

\section{DISCUSSION}

The different analyses described in the previous paragraphs allows a few final considerations to be highlighted. It was possible to exploit different approaches for the acquisition and processing of the data acquired with a commercial UAV RTK multi-rotor platform and evaluate the achievable metric accuracy using different criteria. Three out of the five available acquisitions modes have been tested and analysed, ranging from the acquisition phase to the validation of the results of a direct georeferencing strategy. The PPK approach has been tested exploiting as reference data a CORS station close to the test site. This approach allows to skip the positioning of a second GNSS receiver in the field (of course only in areas with a suitable coverage of the CORS network). The possibility to directly connect the UAV to NTRIP to exploit a NRTK approach has been assessed as well. The conditions to perform this approach are related to the availability of a good GSM network in the test site. This solution is however the best compromise in terms of time and accuracy, enabling rapid survey operations with very high 3D positional accuracies (few centimeters).

While the NRTK processing is almost straightforward and no GCP are required, the PPK approach still requires some intervention from the operator and some technical skills to manage the whole process, including the positioning and measurement of at least one GCP. This fact is mainly caused by the lack of an embedded software package provided by the platform manufacturer and by the format of the data recorded from the system that are not always easily readable and interpretable. Particular care must also be dedicated to the solution of the I.O. phase of the photogrammetric pipeline. This aspect is particularly true in case of an only nadiral dataset, like the one available for this research. As expected from the desk review results confirmed that a wrong estimation of camera I.O. parameters can lead to significant errors on the positional accuracy, especially with respect to the elevation component.

\section{CONCLUSION AND FURTHER PERSPECTIVES}

The possibility to adopt a direct georeferencing approach for the photogrammetric block eliminating the phases of control points positioning and measuring is definitely promising, however some issues still need to be further investigated and solved. Among the different tests performed in this work it is interesting to underline the potentialities connected with an NRTK approach. In the test case presented it was possible to achieve an overall accuracy of the photogrammetric processing in a range of few centimetres, allowing to guarantee a representational scale of at least 1:500 without the use of GCPs. This approach is subjected to a good network coverage but it can possibly reach also higher precisions with further enhancements, that need to be tested and validated.

As is reported before the camera calibration is still a crucial point and a wrong estimation of I.O. parameters could lead to significant errors in the final model. This part of the whole process needs to be further investigated and solutions that allows a reliable estimation of these parameters need to be investigated. An enhancement could derive from the introduction of oblique images in order to strengthen the geometry of the photogrammetric block and enhance the selfcalibration approach. Another advantage could be the availability of a more robust and precise pre-calibration of the UAV camera. Further tests and new acquisitions are underway with a third part GPS/GNSS receiver and the DJI D-RTK2 antenna for both PPK and RTK approaches. A further interesting possibility is connected with the creation and use of the data derived from a virtual reference station thanks to the options offered from the NTRIP and the CORS network. Finally, the impact of these direct georeferencing approaches on the creation of added value products of the photogrammetric pipeline needs to be investigated with additional tests.

\section{ACKNOWLEDGEMENTS}

The authors would like to thank the Laboratory of Geomatics of the DIATI Department of Politecnico of Turin, and in particular 
Prof. Andrea Lingua and Paolo Maschio for the support in the use of the platform.

The authors would like to thank the Municipality of Caluso for allowing the realisation of the survey. The authors would also like to thank D.Einaudi, J. Tumelero and V. Scolamiero for the support during the survey.

Authors would like to underline that the development of this research was affected by the contingent situation related with the COVID-19 emergency. Part of the research was postponed due to the emergency situation and the impossibility to perform further acquisitions and tests.

The authors disclose that a MOU is in force between the authors' institution and the manufacturer of the UAV used. However, the authors declare that the manufacturer had no role in this study, and no conflict of interest exists.

\section{REFERENCES}

Agüera-Vega, F., Carvajal-Ramírez, F., \& MartínezCarricondo, P. (2017). Assessment of photogrammetric mapping accuracy based on variation ground control points number using unmanned aerial vehicle. Measurement: Journal of the International Measurement Confederation, 98(98), 221227.

Aicardi, I., Chiabrando, F., Grasso, N., Lingua, A., Noardo, F., \& Spanò, A. T. (2016). UAV photogrammetry with oblique images: First analysis on data acquisition and processing. International Archives of the Photogrammetry, Remote Sensing and Spatial Information Sciences - ISPRS Archives, 2016Janua(July), 835-842.

Benassi, F., Dall'Asta, E., Diotri, F., Forlani, G., Morra di Cella, U., Roncella, R., ... Santise, M. (2017). Testing Accuracy and Repeatability of UAV Blocks Oriented with GNSSSupported Aerial Triangulation. Remote Sensing, 9(2), 172.

Bolkas, D. (2019). Assessment of GCP Number and Separation Distance for Small UAS Surveys with and without GNSS-PPK Positioning. Journal of Surveying Engineering, 145(3), 1-17.

Bolognesi, M., Furini, A., Russo, V., Pellegrinelli, A., \& Russo, P. (2015). Testing the low-cost rpas potential in 3D cultural heritage reconstruction. In International Archives of the Photogrammetry, Remote Sensing and Spatial Information Sciences - ISPRS Archives (Vol. 40, pp. 229-235).

Chiabrando, F., Giulio Tonolo, F., \& Lingua, A. (2019). Uav direct georeferencing approach in an emergency mapping context. the 2016 central Italy earthquake case study. In International Archives of the Photogrammetry, Remote Sensing and Spatial Information Sciences - ISPRS Archives (Vol. 42, pp. 247-253).

Chiabrando, F., Lingua, A., Maschio, P., \& Teppati Losè, L. (2017). THE INFLUENCE OF FLIGHT PLANNING AND CAMERA ORIENTATION IN UAVS PHOTOGRAMMETRY. A TEST IN THE AREA OF ROCCA SAN SILVESTRO (LI), TUSCANY. ISPRS - International Archives of the Photogrammetry, Remote Sensing and Spatial Information Sciences, XLII-2/W3(March), 163-170.

Forlani, G., Asta, E. D., Diotri, F., Morra, U., Id, R. R., \& Santise, M. (2018). Quality Assessment of DSMs Produced from UAV Flights Georeferenced with On-Board RTK Positioning.

Gabrlik, P. (2015). The use of direct georeferencing in aerial photogrammetry with micro UAV. In IFAC-PapersOnLine (Vol. 28, pp. 380-385).

Georgopoulos, A., Oikonomou, C., Adamopoulos, E., \& Stathopoulou, E. K. (2016). Evaluating unmanned aerial platforms for cultural heritage large scale mapping. ISPRS International Archives of the Photogrammetry, Remote Sensing and Spatial Information Sciences, XLI-B5, 355-362.

Honkavaara, E. (2002). IN-FLIGHT CAMERA CALIBRATION FOR DIRECT GEOREFERENCING ABSTRACT : r. Int. Arch. Photogramm. Remote Sens. Spat. Inf. Sci, (2000).

Martínez-Carricondo, P., Agüera-Vega, F., Carvajal-Ramírez, F., Mesas-Carrascosa, F. J., García-Ferrer, A., \& Pérez-Porras, F. J. (2018). Assessment of UAV-photogrammetric mapping accuracy based on variation of ground control points. International Journal of Applied Earth Observation and Geoinformation, 72(February), 1-10.

Nesbit, P. R., \& Hugenholtz, C. H. (2019). Enhancing UAVSfM 3D model accuracy in high-relief landscapes by incorporating oblique images. Remote Sensing, 11(3), 239.

Nex, F., \& Remondino, F. (2014). UAV for 3D mapping applications: A review. Applied Geomatics, 6(1), 1-15.

Peppa, M. V, Hall, J., Goodyear, J., \& Mills, J. P. (2019). Photogrammetric assessment and comparison of dji phantom 4 pro and phantom 4 rtk small unmanned aircraft systems. In International Archives of the Photogrammetry, Remote Sensing and Spatial Information Sciences - ISPRS Archives (Vol. 42, pp. 503-509).

Rabah, M., Basiouny, M., Ghanem, E., \& Elhadary, A. (2018). Using RTK and VRS in direct geo-referencing of the UAV imagery. NRIAG Journal of Astronomy and Geophysics, 7(2), $220-226$.

Sauerbier, M., \& Eisenbeiss, H. (2010). Uavs for the Documentation of Archaeological Excavations. Proceedings of the Isprs Commission V Mid-Term Symposium Close Range Image Measurement Techniques, 38(5), 526-531.

Taddia, Y., Stecchi, F., \& Pellegrinelli, A. (2019). Using dji phantom 4 rtk drone for topographic mapping of coastal areas. In International Archives of the Photogrammetry, Remote Sensing and Spatial Information Sciences - ISPRS Archives (Vol. 42, pp. 625-630).

Taddia, Y., Stecchi, F., \& Pellegrinelli, A. (2020). Coastal Mapping using DJI Phantom 4 RTK in Post-Processing Kinematic Mode. Drones, 4(2), 9.

Tahar, K. N. (2013). An evaluation on different number of ground control points in unmanned aerial vehicle photogrammetric block. In International Archives of the Photogrammetry, Remote Sensing and Spatial Information Sciences - ISPRS Archives (Vol. XL-2/W2, pp. 93-98). 